\title{
The effects of treatment on target organ damage in hypertensive disease
}

\author{
Jerome Lowenstein
}

Renal and Hypertension Section, NYU Medical Center, New York, NY 10016, USA.

\section{Introduction}

The mortality rate from malignant hypertension has been dramatically reduced and the morbidity and mortality attributable to essential hypertension have been significantly decreased since the introduction of potent antihypertensive drugs (Mroczek et al., 1969; Gudbrandsson et al., 1970; Veterans Administration Cooperative Group, 1970). While the efficacy of antihypertensive treatment now seems quite well established, it has become clear that not all of the target-organ effects of hypertensive disease are equally well prevented or reversed by therapy. This review will attempt to examine some of the responses of target organs to treatment in patients with hypertensive disease.

The major clinical features of malignant or accelerated hypertension - hypertensive encephalopthy, cerebral haemorrhage, and renal failure - can best be viewed as manifestation of a failure of autoregulation or 'autoregulatory breakthrough', i.e., the endorgan damage is a consequence of increased hydrostatic pressure in the distal arteriolar and capillary beds.

\section{The cerebral circulation}

The phenomenon of blood flow autoregulation has been well studied in the cerebral circulation where it has been shown that increased perfusion pressure is paralleled by an increase in vascular resistance and, as a consequence, both organ blood flow and microvascular pressures are maintained at near normal values (Strandgaard et al., 1974). Studies in experimental animals suggest that hypertensive encephalopathy is due to a failure of autoregulation at the very high levels of arterial blood pressure which characterize malignant hypertension. Hypertensive encephalopathy is a clinical syndrome characterized by altered mental

\section{J. Lowenstein, M.D.}

Received 26 February 1985. state, severe headache, seizures, and focal neurological deficits. Pathological examination of the brain reveals cerebral oedema, petechial haemorrhage, and/or focal ischaemic infarction. It was debated for many years whether the sequence of events leading to hypertensive encephalopathy and cerebral haemorrhage was initiated by focal ischaemia - i.e., hypoperfusion (Meyer et al., 1960) - or by hyperperfusion (Farrer et al., 1976), i.e. autoregulatory breakdown. Byrom (1954) observed that the pial blood vessels of rats with renal artery clip hypertension and hypertensive encephalopathy exhibited alternating areas of constriction and dilatation. He demonstrated in rats with brain dysfunction that the dye, trypan blue, passed across the blood brain barrier and stained the brain tissue. Geise (1964) subsequently demonstrated that the penetration of a macromolecular tracer, colloidal carbon, was localized to areas adjacent to the dilated rather than the constricted segments of the cerebral vascular bed. This issue has been re-examined by Tamaki et al. (1984), utilizing a strain of the Okamoto spontaneous hypertensive rat (SHR), bred for susceptibility to the development of hypertensive encephalopathy and stroke - the stroke-prone SHR. These investigators examined the relationship between disruption of the blood-brain barrier, assessed by leakage of Evan's blue tagged albumin, and regional cerebral blood flow, assessed by the accumulation of radioactive antipyrine (which passes freely across the bloodbrain barrier), in stroke prone-SHR exhibiting neurological abnormalities. They found that local blood flow was increased in areas of the cerebral cortex in which the blood-brain barrier was disrupted (Evan's blue staining with little oedema). The symptoms and minimal gross brain pathology suggest that this is a good experimental model for hypertensive encephalopathy: the findings provide evidence that hyperperfusion is responsible for the pathological changes and associated symptoms. In contrast, local cerebral blood flow was significantly reduced in areas in which disruption of the blood-brain barrier was 
associated with marked focal oedema.They suggested that hyperperfusion with transudation of plasma proteins and fluid into the substance of the brain might be the initiating events in regional cerebral ischaemia and therefore responsible for stroke, both ischaemic and haemorrhagic, in these stroke prone SHR.

A pathogenetic role for increased blood pressure and 'autoregulatory breakthrough' in hypertensive encephalopathy was further supported by the finding that unilateral ablation of the cervical sympathetic ganglion, denervating the blood vessels to one side of the brain, resulted in a striking preponderance of focal haemorrhagic and ischaemic infarctions as well as pathological vascular changes in the denervated hemisphere (Sadoshima et al., 1981). Morphometric study of the small cerebral vessels revealed a significant reduction in the wall-to-lumen ratio of vessels on the denervated side, i.e., less arteriolar hypertrophy, suggesting a trophic role for the sympathetic nerves in mediating vessel hypertrophy in the hypertensive animal. Further, the localization of haemorrhagic and ischaemic infarction in the denervated hemisphere lends support to the concept that arteriolar resistance plays an important role in protecting the microvasculature from the damaging effects of increased hydrostatic pressure. This experimental finding may provide an explanation for the clinical observation that hypertensive encephalopathy typically occurs at lower levels of blood pressure in patients with the sudden onset of severe hypertension, e.g., eclampsia or glomerulonephritis, in which arteriolar hypertrophy may not yet have developed.

While the finding of regional cerebral hyperperfusion provides a basis for the observed beneficial effect of antihypertensive therapy in hypertensive encephalopathy and cerebral haemorrhage in man, the findings in the stroke-prone SHR also suggest a mechanism which might account for the protective effect of antihypertensive treatment on the development of ischaemic cerebral infarction in treated patients with benign essential hypertension. The vessel abnormalities responsible for cerebral infarction in the SHR are confined to arteries and arterioles of the order of 50 to 200 microns in diameter. The pathological changes in such vessels in hypertensives, well described in a classic paper by Feigen \& Prose (1959), are not atherosclerotic but rather are described as lypohyalinosis or hypertensive fibrinoid necrosis. It has been suggested that these vascular lesions might be responsible for the small deep lacunar infarcts which account for $20-50 \%$ of non-embolic strokes in hypertensives (Fisher, 1969). While atherosclerosis of larger intracranial vessels is increased in hypertensives and atherosclerosis of these larger arteries correlates with the frequency of lacunar infarcts, localization of the lesions at autopsy or by computerized axial tomography suggest that smaller penetrating vessels are often the site of occlusion. The finding that antihypertensive therapy significantly reduces the incidence of both haemorrhagic and ischaemic cerebral infarcts (Reportof the Management Committee: The Australian Therapeutic Trial in Mild Hypertension, 1980; Hyper $\stackrel{\text { ? }}{+}$ tension Detection and Followup Progran? Cooperative Group, 1979) is more likely attributable to the prevention of hypertensive changes in smalf vessels than to the prevention of cerebral atheros $\unrhd$ clerosis.

\section{The renal circulation}

The pattern of renal vascular involvement in malig을 nant hypertension has changed strikingly in the 2 . years since the introduction of potent antihypertensive? drugs. In the era before effective antihypertensive therapy, the typical renal lesion seen in patients dyingo of malignant hypertension was fibrinoid arteriolitis characterized by the insudation of plasma proteins and fibrin in the walls of preglomerular arterioles? With the advent of potent antihypertensive drugs, the course of the disease has been prolonged (Mroczek $e \mathbb{P}$ al., 1969; Gudbrandsson et al., 1979) and the renaf lesion, seen in biopsy samples, nephrectomy oz necropsy material, is that of marked medial hyperteo. phy and subintimal fibroplasia of arcuate and terlobular arteries associated with a variable degree 050 glomerular sclerosis (Jones, 1974). A pati physiological sequence similar to that which has beeno described in the cerebral circulation, initiated bys hyperfusion and autoregulatory failure, may be responsible for these vascular changes and for the rena年 manifestations in malignant hypertension $-\Omega$ haematuria, proteinuria, and renal failure. Both the arteriolar and small arterial lesions may be the end $\mathrm{B}$ result of exposure of the intrarenal vasculature to increased hydrostatic pressure (Helmchen et al., 1984)? Intrarenal pressure has been found to be elevated in small arteries, arterioles, and glomerular capillaries in several experimental models of hypertension. Koch $e \bar{S}_{3}$ al. (1968) first observed a striking increase in in-trarenal pressure (peritubular capillary pressure) andô glomerular hydrostatic pressure following acute elevation of arterial pressure by carotid artery ligation in the rat; pathological changes in the renal vasculature were not examined. Azar et al. (1974) observed thate. intrarenal pressures were significantly increased in an model of hypertension produced by salt loading, with or without uninephrectomy, in the Holtzman strain of rat. Glomerular pressure was also elevated. Theyw found that single nephron blood flow and filtration rate (SNGFR) were similarly increased. These ratso developed extensive glomerulosclerosis and, as aळ result, overall glomerular filtration rate (GFR) was ${ }^{\circ}$ reduced despite increased single nephron blood flow, $\frac{0}{0}$ 
filtration pressure, and SNGFR, i.e., nephron hyperperfusion.

We observed a similar pattern of increased intrarenal pressure in patients with essential hypertension (Lowenstein et al., 1970). In these studies, peritubular capillary pressure was estimated from measurements of wedged renal vein pressure (WRVP) and, taken together with clearance measurements of GFR and renal plasma flow, were used to estimate glomerular capillary pressure. Patients with moderate hypertension demonstrated significant elevations of intrarenal and glomerular pressure. We attributed the maintenance of normal GFR, in the face of increased glomerular pressure, to the associated increase in proximal tubular hydrostatic pressure with resultant unchanged net filtration pressure. It seems equally possible that the total nephron number was reduced (by glomerulosclerosis) and that hyperfiltration in remaining nephrons offset the loss to maintain a normal overall filtration rate. There is increasing experimental evidence that high glomerular pressure and blood flow may result in glomerular injury leading to glomerular sclerosis (Hostetter et al., 1981). Even the vascular changes, seen as medial smooth muscle hyperplasia and subintimal fibroplasia may reflect the effects of pressure-mediated injury. In tissue culture, vascular smooth muscle hypertrophy can be stimulated by platelet derived growth factor (PDGF)a polypeptide with structural homology to a recognized oncogene sequence - which binds to specific receptors on smooth muscle cells and fibroblasts and initiates DNA synthesis (Schwartz, 1984). It might be speculated that PDGF gains access to muscle cells and subintimal fibroblasts at sites of haemodynamically mediated endothelial injury. While there is good evidence that antihypertensive treatment appears to prevent or slow the rate of progression or renal failure in patients with malignant hypertension, particularly those in whom therapy is begun before glomerular filtration is significantly reduced, slowly progressive renal failure is often observed despite adequate blood pressure control. Experimental studies (Dworkin et al., 1984) suggest that glomerular hyperperfusion may persist despite blood pressure reduction and might be responsible for progressive renal injury. Further studies and clinical correlation will be required to determine whether the specific intrarenal haemodynamic effects of various classes of antihypertensive agents differ and, if so, whether such differences are related to the progression of renal failure in treated patients with malignant hypertension.

\section{Left ventricular hypertrophy}

Left ventricular hypertrophy (LVH) is a well recognized consequence of sustained arterial hypertension.
While it might be expected that the occurrence and severity of left ventricular hypertrophy would be closely related to the magnitude and duration of increased arterial pressure, the degree of LVH, whether assessed by electrocardiographic (ECG) criteria or by direct measurement at necropsy, correlates poorly with mean arterial pressure (Tarazi \& Levy, 1982). Echocardiography has made it possible to examine the relationship of left ventricular mass to left ventricular afterload. Afterload is the total force against which the myocardium must contract during the ejection phase of systole. It follows that systolic blood pressure is probably more important than either diastolic or mean arterial blood pressure as a determinant of hypertrophy. Abi-Samra et al. (1983) examined the relationship between left ventricular wall mass, measured by $\mathbf{M}$ mode echocardiography and systolic blood pressure, and found only a weak correlation. Although poorly understood, it appears that other factors such as the inotropic state, the degree of adrenergic stimulation and perhaps the activity of the renin-angiotensin system may be important determinants of the degree to which the myocardium hypertrophies in response to any given level of blood pressure. Sen (1983) found that regression of myocardial hypertrophy, in the spontaneous hypertensive rat, followed treatment with either methyldopa, a combination of reserpine, hydralazine and thiazide, or captopril, but not treatment with the vasodilators, hydralazine or minoxidil, despite more effective blood pressure lowering with the latter drugs. She postulated that sympathetic inhibition by methyldopa or reserpine, as contrasted with reflex sympathetic stimulation associated with hydralazine or minoxidil, might account for the disparate effects on the regression of myocardial hypertrophy. Lundin \& Hallback-Norlander (1984) compared the effects of methyldopa, metoprolol, and the calcium channel antagonist, felopidine, on regression of cardiovascular changes in the SHR and concluded that the extent of reduction in arterial pressure was the major determinant of the extent of reversal of structural changes.

The application of echocardiography to the study of myocardial hypertrophy in hypertensive disease has yielded several interesting and surprising findings. First, it has been noted that left ventricular hypertrophy may be asymmetric rather than concentric in hypertensive disease. Hypertrophy has been found in young relatively mild hypertensives (Tarazi \& Levy, 1982). Hypertrophy has been observed to regress during antihypertensive therapy in man. Wollam et al. (1983) made serial echocardiographic measurements of left ventricular posterior wall thickness and septal thickness in a small series of hypertensive patient treated for 18 months with either methyldopa, hydrochlorothiazide, or a combination of these agents. Despite comparable blood pressure reduction with the 
three treatment protocols, regression of hypertrophy was not observed when the thiazide was given alone. The important implication of these early experimental and clinical studies appears to be that left ventricular hypertrophy in hypertension is not a simple function of the degree of blood pressure elevation and, as a corollary, that antihypertensive drugs might differ in their capacity to prevent or to cause regression of left ventricular hypertrophy (Tarazi \& Fouad, 1984).

\section{Coronary heart disease}

Finally, I would like to consider the effects of antihypertensive therapy on the develpment and course of coronary heart disease. Epidemiological studies have demonstrated that hypertension is a major risk factor for the development of coronary heart disease. With effective control of blood pressure in severe hypertension, heart failure, hypertensive encephalopathy, and renal failure have become less common and coronary heart disease and stroke are emerging as the major cardiovascular consequences of mild hypertension (defined as diastolic pressure between 90 and $114 \mathrm{~mm} \mathrm{Hg}$ ). Data from the Framingham study (Castelli, 1984) indicate that for adults with blood pressure greater than $160 / 95 \mathrm{~mm} \mathrm{Hg}$ the risk of coronary heart disease (CHD) is increased 2-3-fold and that of stroke 7-fold; for patients with BP 140-159/ $90-94 \mathrm{~mm} \mathrm{Hg}$, CHD risk is increased 1.5-fold and stroke 3-fold (Kannel, 1977).

The Veterans Administration Cooperative study of antihypertensive therapy (1970) found that in the stratum of patients with diastolic blood pressures from 90-114 $\mathrm{mm} \mathrm{Hg}$ the effects of lowering blood pressure were limited to reduction in the frequency of accelerated hypertension, stroke and congestive heart failure; the incidence of myocardial infarction was not reduced in the actively treated patients. It might be argued that, as the average age of the men in the study was $51 \mathrm{y}$, coronary atherosclerosis was probably already well established at the time therapy was begun, and that it was therefore not surprising that the incidence of myocardial infarction was not reduced by antihypertensive therapy.

The next major placebo-controlled study of the effects of antihypertensive therapy was the US Public Health Service Hospitals Cooperative Study (Smith, 1977). This $10 \mathrm{y}$ trial in 389 men and women, average age $44 \mathrm{y}$, with diastolic BP $90-114 \mathrm{~mm} \mathrm{Hg}$, excluded patients with either cardiomegaly or abnormal resting or exercise ECG. While all morbid events were reduced by almost $60 \%$ in the treated patients, protection was largely limited to the category of 'hypertensive' complications (stroke, LVH, LV strain, and heart failure). The incidence of myocardial infarction was not reduced in the treated group. Two additional placebo controlled studies have addressed $\stackrel{2}{3}$ this issue; the Australian Therapeutic Trial in Mild $\stackrel{\mathbb{Q}}{\varrho}$ Hypertension studied 3400 hypertensive patients $C$ (1980) and the Oslo Study 785 men (Helgeland, 1980). Both trials confirmed the overall benefit of blood $\stackrel{5}{+}$ pressure reduction but failed to find a significant $\mathrm{C}$ reduction in the incidence of myocardial infarction $\frac{\overline{\bar{c}}}{\overline{\mathrm{s}}}$ with antihypertensive drug treatment.

Consideration of the possible reasons why anti- $\stackrel{\mathbb{D}}{\circ}$ hypertensive therapy has failed to reduce the incidence of myocardial infarction raises several possibilities: (1) $\vec{\circ}$ The possibility that atherosclerosis and hypertension 'have a common cause but are not causally related' $\vec{\omega}$ seems to ignore the many experimental studies which demonstrate a causal role for hypertension in theo genesis of atherosclerosis and fails to explain the increased incidence of atherosclerosis in secondary? forms of hypertension. (2) The presence of coronary atherosclerosis in young men was demonstrated în. autopsy series in the Korean War (Enos et al., 1955) and attests to the early onset of this lesion. The possibility that this lesion was already present and that ${ }^{\circ}$ its course could not be changed by antihypertensive $\vec{C}$ therapy cannot be rigorously excluded. (3) With the development of methods for ambulatory blood pres- $\frac{\overrightarrow{\mathbb{D}}}{\mathbb{D}}$ sure monitoring, it has become clear that blood 3 pressure control in treated hypertensives does not approximate the control of blood pressure over the $\overrightarrow{0}$ course of $24 \mathrm{~h}$ in normotensive individuals. If athera- $\infty$ sclerosis is initiated by small areas of intimal injus or which allow the access of growth factors and lipoproteins to subintimal smooth muscle cells, it may be that the amplitude of blood pressure deviationso rather than the average or resting blood pressure may be the critical variable in determining the development $\stackrel{\mathbb{}}{2}$ of atherosclerotic lesions. The importance of $\overrightarrow{\vec{O}}$ haemodynamic factors other than average blood 3 pressure is evident from the unique localization of atherosclerotic plaques at specific sites (branch points, vessel ostia) in the arterial tree.

Finally, it has recently been recognized that many of the antihypertensive drugs affect serum lipoprotein $\frac{3}{3}$ concentrations. It may be speculated that drug-induced alterations in lipoprotein and cholesterol $\stackrel{\circ}{\circ}$ metabolism could offset the potentially beneficial effects of blood pressure reduction. Diuretics, both thiazides and chlorthalidone, have been found to $D$ induce an increase in low density lipoprotein choles-을 terol concentration (Goldman et al. 1980; Ballantyne N $\&$ Ballantyne, 1983). Some investigators have reported ${ }^{\circ}$ that beta-adrenergic blocking drugs induce a decrease $\mathcal{N}$ in the concentration of high density lipoprotein $\mathrm{W}$ cholesterol (Leren et al., 1980; Waal-Manning \& Simpson, 1977). In contrast to the effects of betaadrenergic antagonists the selective alpha-adrenergic $\Phi_{\overparen{D}}$ antagonist, prazosin, has been reported to reduce ${ }^{?}$ cholesterol and triglycerides and to cause a modest ${ }^{\circ}$ 
increase in the concentration of high density lipoprotein cholesterol (Leren et al., 1980; Lowenstein \& Neusby, 1984).

The importance of serum cholesterol concentration as a risk factor for the development of coronary heart disease is well established. Low density lipoprotein cholesterol concentration is positively correlated with coronary risk and high density lipoprotein cholesterol is negatively correlated (Castelli, 1984). In light of the importance of serum cholesterol concentration and lipoprotein fractions in the pathogenesis of coronary artery disease, the finding that diuretic agents and

\section{References}

ABI-SAMRA, F., FOUAD, F.M. \& TARAZI, R.C. (1983). Determinants of left ventricular hypertrophy and function in hypertensive patients. American Journal of Medicine, (Suppl Sept.), 26.

AZAR, S., TOBIAN, L. \& JOHNSON, M.A. (1974). Glomerular, efferent, arteriolar peritubular capillary and tubular pressures in hypertension. American Journal of Physiology, 227, 1045.

BALLANTYNE, D. \& BALLANTYNE, F.C. (1983). Thiazides, beta blockers and lipoproteins. Postgraduate Medical Journal, 59, 483.

BYROM, F.B. (1954). The pathogenesis of hypertensive encephalopathy and its relation to the malignant phase of hypertension. Lancet, ii, 201.

CASTELLI, W.P. (1984). Epidemiology of coronary heart disease: the Framingham Study. American Journal of Medicine, 76, (Suppl 2A), 4.

DWORKIN, L.D., FEINER, H.D. \& RANDAZZO, J. (1984). Evidence for haemodynamically mediated glomerular injury despite antihypertensive therapy in rats with desoxycorticosterone salt (DOC-salt) hypertension (ABS). Proceedings of 17th Annual Meeting of the American Society of Nephrology, 91A.

ENOS, W.F.Jr., BEYER, J.C. \& HOLMES, R.H. (1955) Pathogenesis of coronary artery disease in American soldiers killed in Korea. Journal of the American Medical Association, 158, 912.

FARRAR, J.K., JONES, J.J., GRAHAM, D.I., STRANDGAARD, S. \& MACKENZIE, E.T. (1976). Evidence against cerebral vasopasm during acutely induced hypertension. Brain Research, 104, 176.

FEIGEN, E. \& PROSE, P. (1959). Hypertensive fibrinoid arteritis of the brain and gross cerebral haemorrhage. A form of 'hyalinosis'. Archives of Neurology, 1, 98.

FISHER, C.M. (1969). The arterial lesion underlying lacunes. Acta Neuropathologica, 12, 1.

GEISE, J. (1964). Acute hypertensive vascular disease. 2. Studies on vascular reaction patterns and permeability changes by means of vital microscopy and colloidal tracer technique. Acta Pathologica et Microbiologica Scandinavica, 62, 49.

GOLDMAN, A.I., STEEL, B.W., SCHNAPER, H.W., FITZ, A.E., FROLICH, E.D. \& PERRY, H.M. (1980). Serum lipoprotein levels during chlorthalidone therapy. Journal of the American Medical Association, 244, 1691. adrenergic blocking drugs alter serum lipid concentrations raises several important questions. (1) How long do these serum lipid changes persist? Present data suggests that they are still evident after at least 1 year of treatment with diuretic (Goldman et al., 1980). The duration of the changes induced by alpha- and betaadrenergic antagonists is not known beyond about 2 months. (2) Are the changes quantitatively sufficient to influence coronary risk? (3) Do these drug-induced alterations in lipoprotein cholesterol concentrations change coronary risk? The answers to these important questions are not yet at hand.
GUDBRANDSSON, T. HANSON, L., HERLITZ, H. \& ANDREN, L. (1979). Malignant hypertension - improving prognosis in a rare disease. Acta Medica Scandinavica, 206, 495.

HELGELAND, A. (1980). Treatment of mild hypertension: a five year controlled drug trial: the Oslo Study. American Journal of Medicine, 69, 725.

HELMCHEN, U., BOHLE, R.M., KNIESSLER, U. \& GROENE, H.J. (1984). Intrarenal arteries in rats with early two-kidney one clip hypertension. Hypertension, 6, (Suppl III) 87.

HOSTETTER, R.H., OLSEN. J.L., RENNKE, H.G., VENKATACHALAM, M.A. \& BRENNER, B.M. (1981). Hyperfiltration in remnant nephrons: a potentially adverse response to renal ablation. American Journal of Physiology, 241, 85.

Hypertension Detection and Follow-up Program Cooperative Group (1979). Journal of the American Medical Association, 242, 2563.

JONES, D.B. (1974). Arterial and glomerular lesions associated with severe hypertension. Laboratory Investigation, 31, 303.

KANNEL, W.M. (1977). Importance of hypertension as a major risk factor in cardiovascular disease. In Hypertension, Genest J, Koiw, E. \& Kuchel, O. (eds). p. 888. McGraw-Hill: New York.

KOCH, K.M., AYNEDJIAN, H.S. \& BANK, N. (1968). Effect of acute hypertension on sodium reabsorption by the proximal tubule. Journal of Clinical Investigation, 47, 1696.

LEREN, P., FOSS, P.O., HELGELAND, A., HJERMANN, I., HOLME, I. \& LUND-LARSON, P.G. (1980). Effect of propranolol and prazosin on blood lipids. The Oslo Study. Lancet, ii, 4.

LOWENSTEIN, J., BERANBAUM, E.R., CHASIS, H. \& BALDWIN, D.S. (1970). Intrarenal pressure and exaggerated natriuresis in essential hypertension. Clinical Science, 38, 359.

LOWENSTEIN, J. \& NEUSBY, A.-J. (1984). Effects of prazosin and propranolol on serum lipids in patients with essential hypertension. American Journal of Medicine, 76, (Suppl 2A) 79 .

LUNDIN, S.A. \& HALLBACK-NORLANDER, M.I.L. (1984) Regression of structural cardiovascular changes by antihypertensive therapy in spontaneously hypertensive rats. Journal of Hypertension. 2, 11.

MEYER, J.A., WALTZ, A.G. \& GOTOH, F. (1960). Pathogenesis of cerebral vasospasm in hypertensive encephalopathy. I. 
Effects of acute increases in intraluminal blood presssure on pial blood flow. Neurology, 10, 735.

MROCZEK, W.J., DAVIDOV, M.E., GAVRILOVICH, L. \& FINNERTY, F.A. (1969). The value of aggressive therapy in the hypertensive patient with azotemia. Circulation, 40, 893.

SADOSHIMA, S., BUSIJA, D., BRODY, M. \& HEISTADT, D. (1981). Sympathetic nerves protect against stroke in stroke-prone spontaneously hypertensive rats. Hypertension, 3, (Suppl I), 1-124.

SCHWARTZ, S.M. (1984). Smooth muscle proliferation in hypertension. Hypertension, 6, (Suppl I), 1-56.

SEN, S. (1983). Regression of cardiac hypertrophy: experimental animal model. American Journal of Medicine, (Suppl Sept.), 87.

SMITH, W.M. (1977). Treatment of mild hypertension: results of a ten year intervention trial. Circulation Research, 40, (Suppl I), 98.

STRANDGAARD, S., MACKENZIE, E.T., SENGUPTA, D., ROWAN, J.O., LASSEN, N.A. \& HARPER, A.M. (1974). Upper limit of autoregulation of cerebral blood flow in the baboon. Circulation Research, 34, 435.

TAMAKI, K., SADOSHIMA, S., BAUMBACK, G.L.K.,
IADECOLA, C., REIS, D.J. \& HEISTADT, D.D. (1984) 2 Evidence that disruption of the blood-brain barrie precedes reduction in cerebral blood flow in hypertensive encephalopathy. Hypertension, 6, (Suppl I), 1-75.

TARAZI, R.C. \& FOUAD, F.M. (1984). Reversal of cardiaes hypertrophy in humans. Hypertension, 6, (Suppl III), $140-$

TARAZI, R.C. \& LEVY, M.N. (1982). Cardiac responses tof increased afterload. Hypertension, 4, (Suppl II), 8.

The Australian Therapeutic Trial in Mild Hypertension? (1980). Lancet, i, 1261.

Veterans Administration Cooperative Study Group on Anti⿱ hypertensive Agents. (1970). Effects of treatment onmorbidity in hypertension - results in patients with diastolic blood pressure averaging 90 through $114 \mathrm{~mm} \mathrm{Hg} \overrightarrow{\vec{\omega}}$ Journal of the American Medical Association, 213, 1143.

WAAL-MANNING, H. \& SIMPSON, F.O. (1977). Betablockers and lipid metabolism. British Medical Journal, 2, 705.

WOLLAM, G.L., HALL, W.D., PORTER, V.D., DOUGLAS: M.B., UNGER, D.J., BLUMENSTEIN, B.A., COTSONIS, G.A., KNUDTSON, G.A., FELNER, J.M. \& SCHLANT, R.C. (1983) Time course of regression of left ventricular hypertrophy in treated hypertensive patients. American Journal of
Medicine, (Suppl Sept.) 100 . 